\title{
How accurately do routinely reported HIV viral load suppression proportions reflect progress towards the 90-90-90 target in the population on antiretroviral treatment in Khayelitsha, South Africa?
}

\author{
J Euvrard, ${ }^{1}$ MA, MPH; T Schulz,${ }^{2}$ MB ChB; K Hilderbrand, ${ }^{1}$ MSc; M Bosland, ${ }^{1}$ BPubAdmin Hons; M Osler, ${ }^{1}$ MPH; \\ A Boulle, ${ }^{1,2} \mathrm{MB} \mathrm{ChB}, \mathrm{PhD} ;$ M-A Davies, ${ }^{1} \mathrm{MB} \mathrm{ChB}, \mathrm{PhD}$ \\ ${ }^{1}$ Centre for Infectious Disease Epidemiology and Research, Department of Public Health and Family Medicine, Faculty of Health Sciences, \\ University of Cape Town, South Africa \\ ${ }^{2}$ Department of Health, Provincial Government of the Western Cape, Cape Town, South Africa
}

Corresponding author: J Euvrard (jonathan.euvrard@uct.ac.za)

\begin{abstract}
Background. The Joint United Nations Programme on HIV/AIDS (UNAIDS) third 90-90-90 target requires 90\% of patients on antiretroviral treatment (ART) to be virally suppressed $(<1000$ copies $/ \mathrm{mL}$ ). In Khayelitsha, Cape Town, South Africa viral load (VL) suppression of $<400$ copies $/ \mathrm{mL}$ was reported as $89 \%$ in 2016 , but only $56 \%$ of patients had a result recorded in routine data. We conceived a VL 'cascade' to represent the steps required for an expected VL to be reported as complete in routine data and thus contribute to reported VL suppression: among those for whom a VL is 'expected', a sample must be collected and tested ('done'), a result must be 'filed' in the patient folder, 'noted' by a clinician and electronically 'captured.' The low reported completion suggested gaps along the VL cascade and cast doubt on the validity of reported suppression.

Objectives. To assess the validity of routinely reported VL suppression and identify barriers to VL completion.

Methods. A retrospective cohort study between 1 July 2015 and 30 June 2016, which included all Khayelitsha patients receiving ART, with a routine VL expected, was conducted. We obtained data routinely captured on site and VL data from the laboratory system. A sample of 1035 patient folders was reviewed. VL suppression was calculated using laboratory data, including all tests done, and compared with reported suppression based on on-site captured electronic data. Successful progression through each step on the VL cascade was estimated. We used logistic regression to identify factors associated with laboratory data and reported VL testing.

Results. Of 22991 patients for whom a routine VL test was due, 84\% were done, 79\% filed, $76 \%$ noted and 55\% captured. Using all laboratory data, VL suppression was estimated as $82 \%, 87 \%, 89 \%$ and $91 \%$ at the $50,200,400$ and 1000 copies/mL thresholds, respectively, but reported suppression using captured results was $80 \%, 86 \%, 88 \%$ and $89 \%$ at those thresholds. Routine VL testing is more likely to be done in children $<15$ years old (adjusted odds ratio (aOR) 1.89, 95\% confidence interval (CI) $1.45-2.48$ ) and pregnant women (aOR 1.90 , 95\% CI 1.28 - 2.81) than in men, adjusted for facility.

Conclusions. Despite a low reported completion, VL testing completion was high. Reported suppression using captured data was similar to suppression calculated using all laboratory data, which provided an accurate measure of progress towards the 90-90-90 target. More work is needed to reach the $16 \%$ of patients missed by routine testing.
\end{abstract}

S Afr Med J 2019;109(3):174-177. DOI:10.7196/SAMJ.2019.v109i3.13456

The Joint United Nations Programme on HIV/AIDS (UNAIDS) 90-90-90 strategy ambitiously aspires to have $90 \%$ of people living with HIV (PLWH) know their status, 90\% to receive sustained antiretroviral treatment (ART) and $90 \%$ to achieve viral load (VL) suppression by $2020 .{ }^{[1]}$ Many studies in different settings have assessed progress compared with these targets, with significant heterogeneity in data sources, outcome definitions, methods and results. ${ }^{[2]}$

The Khayelitsha cohort in Cape Town, South Africa (SA) is one of the oldest and largest HIV cohorts in the country. Free public sector ART was initially rolled out in SA in 2001, in partnership with Médecins Sans Frontières. ${ }^{[3]}$ VL testing is the gold standard in HIV treatment monitoring. ${ }^{[4,5]}$ Unusually for a low-resource setting, VL monitoring has been widely used in Khayelitsha since programme inception in 2001. With 22000 patients receiving ART and in care at provincial facilities in 2016, Khayelitsha reported almost $89 \%$ suppression in quarterly reports; however, there was only $56 \%$ completion of routine
VL tests indicated by national guidelines (R Holtman, Western Cape Department of Health - personal communication, 2017). This low reported completion does not necessarily imply low actual completion, as it may be the result of incompleteness in routine data capturing.

For this study, the concept of a VL 'cascade' was introduced to represent the steps required for an 'expected' VL to be reported as complete in routine data and thus contribute to reported VL suppression in routine quarterly reports (Fig. 1). As a first step, a blood sample was taken in patients in whom a routine VL was expected (step 1). This blood sample must then be processed by the laboratory ('done') (step 2), the test result printed, couriered to the facility, sorted and distributed to the appropriate registry and 'filed' by a clerk in the patient folder (step 3), the result 'noted' by a clinician in the visit summary paper stationery at the next clinical assessment (step 4) and electronically 'captured' by a clerk onto the primary healthcare infor- 
mation system (PHCIS) (step 5). Finally, as routine quarterly ART data report on facility-based ART-naive cohorts, VL results for patients who initiated outside the facility at which they were being cared for were not included in reports (step 6).

The low reported completion for Khayelitsha in 2016 therefore raised two important questions: was the high reported suppression proportion a valid measure of progress towards the third 90 , and what were the barriers to testing and recording of VL results in the context of widely available laboratory testing? This study aimed to answer these questions by describing a VL cascade from expected to reported, and estimating success and failure at each step on the cascade.

\section{Methods \\ Setting}

The Khayelitsha cohort has been described in detail elsewhere. ${ }^{[6]}$ Briefly, Khayelitsha is the largest township in Cape Town, with a high burden of HIV. The provincial government initiated the provision of free ART services at primary healthcare facilities in
$2001 .^{[3]}$ Eligibility criteria have evolved in response to World Health Organization (WHO) recommendations, with universal test and treat adopted nationally in September 2016. ${ }^{[7]}$

\section{Study design}

A retrospective cohort was constructed, including all patients on ART and in care at provincial healthcare facilities in Khayelitsha, with a routine VL expected during 1 July 2015 - 30 June 2016.

\section{Population}

In the ART programme in SA, routine quarterly reports follow naive cohorts while in care at their initiating facility. Patients who die, transfer out or are lost to follow-up (defined as 90 days without medication in hand) exit the cohort. Even if they return to care elsewhere, they are no longer included in the reported suppression proportion. By contrast, the intended denominator for the 'third 90' includes all patients receiving ART, regardless of where they access care. This study included all patients receiving ART in Khayelitsha during the study period,

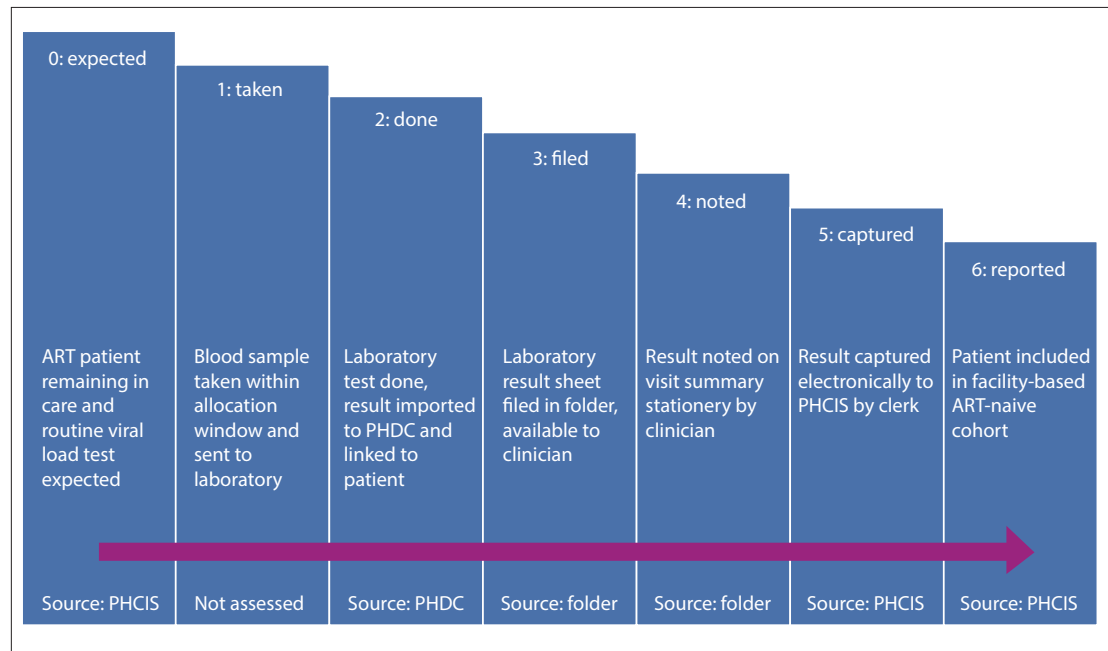

Fig. 1. Viral load cascade - from expected to reported. $(A R T$ = antiretroviral treatment; $P H D C=$ Provincial Health Data Centre; PHCIS = primary healthcare information system.)

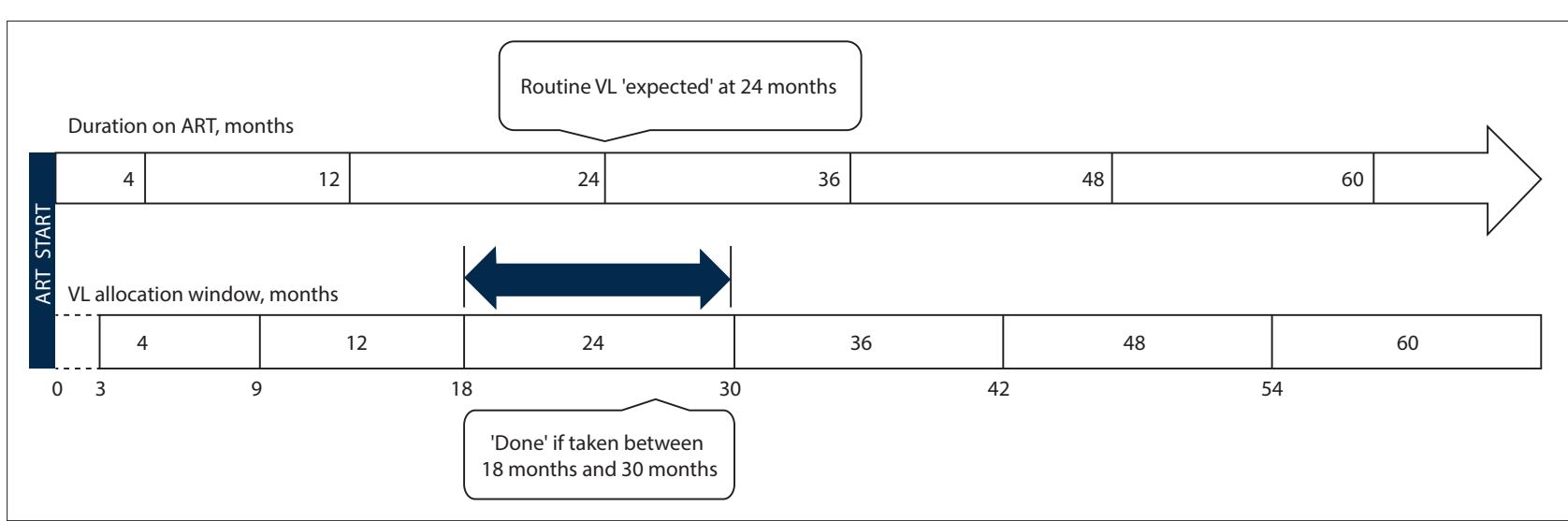

Fig. 2. Viral load allocation, using a 24-month due date as an example. $(V L=$ viral load; $A R T=$ antiretroviral treatment. $)$ 
the cascade, data were requested from PHCIS and PHDC. PHCIS contains routine data digitised by clerks on site. PHDC contains all data from the National Health Laboratory Service (NHLS), which performs all VL testing for patients in care at provincial facilities in Khayelitsha. All VL testing during the study period was performed by the NHLS using an Ampliprep/COBAS TaqMan HIV-1 Test v2 (Roche, Switzerland). Step 1 could not be directly assessed, e.g. if a test was not registered by the laboratory system owing to incorrect or illegible request forms, it would not be counted as done at step 2, despite a blood sample having been taken within the window period and sent to the laboratory. Completion at steps 3 and 4 was assessed by physical folder review, for which folders were drawn on site at facilities and some data captured directly to a stand-alone encrypted electronic database. Routine patient data in all sources (physical folder, PHCIS, NHLS and PHDC) were captured and linked with the unique patient identifier used across the public health platform in Western Cape Province. ${ }^{[10]}$

\section{Data analysis}

The VL cascade is simplified insofar as it reports the furthest step a VL reached on the cascade and assumes that the VL successfully reached all previous steps. Only VLs done (step 2) but not captured (step 5) were sampled for physical folder review. The folder review then generated proportions of the sample, which were filed (step 3) and noted (step 4). These proportions (of the sample) could then be added to the proportion captured (of the total) to generate the proportions filed and noted on the cascade.

Statistical analyses were performed using Stata 14 (StataCorp., USA). The sample of folders selected for review was prepared using the simple random sample function, clustered on healthcare facility.

A logistic regression model was built to investigate the effect of facility, age category and pregnancy status on VL testing completion (done) and inclusion in routine reports (reported). Healthcare facility was included to account for different operational practices at different facilities. Age category and pregnancy status were included because different models of care are provided for children and pregnant women and different clinical guidelines are followed. Adjusted odds ratios (aORs) with 95\% confidence intervals (CIs) were estimated using this model.

\section{Ethical approval}

The research was approved by the Faculty of Health Sciences Human Research Ethics Committee at the University of Cape Town (ref. no. HREC: 270/2017).

\section{Results}

There were 22991 patients receiving ART and in care at provincial healthcare facilities in Khayelitsha between 1 July 2015 and 30 June 2016 with a VL expected, of whom 18450 (84\%) had a VL performed within their window period. Of these, 11100 (60\%) were included in the reported suppression proportion in routine data: $1790(10 \%)$ patients were excluded because they were not part of the naive cohort at the reporting facility, and $5560(30 \%)$ were excluded because they were not captured (step 5). Of those not captured, 1035 physical folders were reviewed, and completion at steps on the cascade was estimated to be $84 \%$ done (VL sample taken and tested), $79 \%$ filed, $76 \%$ noted by a clinician, and $55 \%$ captured electronically.

Actual completion (done) and reported completion (reported) varied by facility, age category and pregnancy status (Table 1). Expected VLs were less than half as likely to be done at facility $\mathrm{C}$ than at facility A (aOR 0.46, 95\% CI 0.42 - 0.51). Expected VLs at facility C were also less likely to be included in the reported suppression proportion
(aOR $0.74,95 \%$ CI 0.69 - 0.78). Children $<15$ years of age were more likely than men to have a routine VL done (aOR 1.89, 95\% CI 1.45 2.48). Despite pregnant women being almost twice as likely as men to have a VL test done within the routine window period, their results were 0.60 times as likely to be included in reported suppression proportions (aOR 1.90, 95\% CI $1.28-2.81$ and aOR 0.60, 95\% CI 0.47 - 0.77, respectively). The inclusion of duration on ART in the model made no meaningful difference to the effect estimates in the model (Supplementary Table 1).*

Suppressed VLs of $<400$ copies/mL were slightly less likely to be included in reported suppression proportions (aOR 0.70, 95\% CI 0.63 - 0.77). Actual VL suppression among all the VLs done, calculated using laboratory data, was $82 \%, 87 \%, 89 \%$ and $91 \%$ at the $50,200,400$ and 1000 copies $/ \mathrm{mL}$ thresholds, respectively, but reported suppression would have been $80 \%, 86 \%, 88 \%$ and $89 \%$ at those same thresholds.

\section{Discussion}

The reported VL suppression proportion of $<400$ copies $/ \mathrm{mL}$ from routine data (89\%) differed by only $1 \%$ when all VL results from the laboratory were included, despite only $60 \%$ contributing to reported suppression. This confirms that Khayelitsha is very close to achieving the third 90 among those with available results. However, taking into consideration the $16 \%$ of patients not tested, the proportion of patients with confirmed suppression of $<400$ copies $/ \mathrm{mL}$ declined to $75 \%$ of those receiving ART and in care. This is concerning, as it implies that even as we successfully increase ART coverage, the proportion of patients on ART who may be at risk of transmitting HIV remains between $10 \%$ and $25 \%$.

Reported completion proportions were low, mostly owing to failure to test patients (16\%) and failure to capture VL results electronically (21\%). A trial is currently being performed of the inclusion of electronically imported test results from laboratory systems in routine quarterly reports in the Western Cape and would obviate the need for manual capturing of the results into PHCIS. However, the advantage of manual capturing from the folder was that it allowed for indirect monitoring of other steps in the VL cascade, as the presence of a VL result in routine data suggested successful progress through previous steps. Specifically, it implied that the result was available to the clinician at the next clinical assessment, as the clinician had to note it in the stationery for a clerk to capture electronically. Importing results will improve the completeness of data in routine reports, but it will no longer be possible to make inferences regarding intermediate steps on the VL cascade from routine data. Reassuringly, there was relatively little loss on the cascade between VL being done (step 2), filed (step 3) and noted (step 4), implying that results from VL tests that were done were usually available to clinicians at the next clinical assessment.

Our results suggest that if electronic import of test results from laboratory systems is successfully implemented, the reported VL completion proportion will increase substantially, but there will be little effect on the reported suppression proportion. Importantly, this study only assessed whether clinicians noted the VL results in the folder; it did not attempt to assess the effective use of VL results for clinical decision-making. Further research in this important area is required.

The variation in actual completion between facilities based on laboratory data suggests facility-specific challenges that require further investigation. The variation by pregnancy status and age category was expected, as clinical guidelines recommend more frequent testing for pregnant women and children.

The under-representation of pregnant women in the reported suppression proportion may be due to increased mobility during pregnancy because of movement between integrated maternal ART 
Table 1. Patient characteristics, outcomes and adjusted odds ratios

\begin{tabular}{|c|c|c|c|c|c|}
\hline & \multirow{2}{*}{$\begin{array}{l}\text { Expected, } \\
n(\%)\end{array}$} & \multicolumn{2}{|c|}{ Done } & \multicolumn{2}{|c|}{ Reported } \\
\hline & & $n(\%)$ & aOR $(95 \% \mathrm{CI})$ & $n(\%)$ & aOR $(95 \% \mathrm{CI})$ \\
\hline Facility A & $7129(32)$ & $6313(34)$ & 1.00 & $3815(34)$ & 1.00 \\
\hline Facility B & $5916(27)$ & $5130(28)$ & $0.84(0.76-0.94)$ & $3181(29)$ & $1.01(0.94-1.08)$ \\
\hline Facility C & $8946(41)$ & $7007(38)$ & $0.46(0.42-0.51)$ & 4104 (37) & $0.74(0.69-0.78)$ \\
\hline Men & $6097(28)$ & $5012(27)$ & 1.00 & $3138(28)$ & 1.00 \\
\hline Non-pregnant women & $15011(68)$ & $12649(69)$ & $1.17(1.08-1.27)$ & $7546(68)$ & $0.96(0.90-1.01)$ \\
\hline Pregnant women & $275(1)$ & $246(1)$ & $1.90(1.28-2.81)$ & $107(1)$ & $0.60(0.47-0.77)$ \\
\hline Children $<15$ years & $608(3)$ & $543(3)$ & $1.89(1.45-2.48)$ & $309(3)$ & $0.99(0.84-1.17)$ \\
\hline Years on ART, median (IQR) & $4(2-7)$ & $4(2-7)$ & $1.09(1.08-1.11)$ & $4(2-7)$ & $1.07(1.06-1.08)$ \\
\hline
\end{tabular}

clinics and routine ART clinics. ${ }^{[11]}$ As discussed above, routine reports include only facility-specific naive ART cohorts. However, this deserves further investigation, as pregnant and breastfeeding women are vulnerable groups and viral suppression is critical to prevent vertical transmission. ${ }^{[12]}$ Since the conclusion of this study, a separate routine quarterly report specifically for pregnant women was mandated by the national government.

Overall, actual completion was relatively high, but more must be done to close the gaps and reach patients who are not being tested, especially men and non-pregnant women. Suppressed and unsuppressed VL results inform patient management and clinical care. A suppressed VL may be used along with other indications to identify patients for recruitment to differentiated models of care, while an unsuppressed VL alerts a clinician to possible adherence challenges or drug resistance. We noted above that suppressed VL results were slightly less likely to successfully complete the VL cascade than unsuppressed VL results. This might suggest missed opportunities to recruit stable patients to differentiated models of care, thereby unburdening the health facilities. It also suggests that routine data may minimally underestimate the success of the ART programme.

\section{Study limitations and strengths}

This study has limitations. Firstly, the small proportion of requested VLs that were not successfully processed by the laboratory could not be estimated. Secondly, in routine quarterly ART reports, and in the third 90 as defined by UNAIDS, the suppression proportion was calculated at a point in time using a single VL. By contrast, sustained suppression over time is required to reap the full benefits of ART and mitigate the risk of transmission. Thirdly, the results of this study may not be generalisable regarding other settings. Three large provincial facilities were included and, while the main findings are likely to apply to similar facilities in metropolitan areas of SA, some of the results may not be generalisable with regard to settings outside Khayelitsha.

Strengths of this study include the assessment of routine data on $>20000$ patients from multiple facilities, including adults, pregnant women and children. This was made possible by close collaboration with the Provincial Department of Health, and the availability of harmonised data from different data sources in the health system provided by PHDC.

\section{Conclusion}

Despite low reported VL completion, actual VL testing completion was high. The study confirmed the high levels of suppression that are routinely reported. More work is needed to reach the $16 \%$ of patients missed by routine testing. Most VL results were accessed and noted by clinicians, and further research is necessary to assess how effectively these results are used in clinical decision-making.

*Supplementary data. Supplementary Table 1 is available from the corresponding author on request.

\section{Declaration. None.}

Acknowledgements. We thank the Khayelitsha staff and those who helped with data collection: Zama Mshweshwe, Thembekile Mdlalo, Eunice Beneke and Koliswa Konkwane, as well as the PHCIS and PHDC teams.

Author contributions. All authors were involved in conceptualising the study design. JE, TS, KH and MB contributed to data collection. JE conducted the analysis and drafted the original manuscript. TS and MD contributed to the analysis, interpretation of data and critical revision of the scientific content. All authors reviewed and approved the final version of the manuscript.

Funding. None.

Conflicts of interest. None.

1. Joint United Nations Programme on HIV/AIDS (UNAIDS). 90-90-90. An ambitious treatment target to help end the AIDS epidemic. 2014. http://www.unaids.org/sites/default/files/media_asset/90-90-90 en_0.pdf (accessed 21 January 2019).

2. Granich R, Gupta S, Hall I, Aberle-Grasse J, Hader S, Mermin J. Status and methodology of publicly available national HIV care continua and 90-90-90 targets: A systematic review. PLOS Med 2017;14(4):e1002253. https://doi.org/10.1371/journal.pmed.1002253

3. Coetzee D, Hildebrand K, Boulle A. Outcomes after two years of providing antiretroviral treatment in Khayelitsha, South Africa. J Acquir Immune Defic Syndr 2004;18(6):887-895. https://doi.org/10.1097 100002030-200404090-00006

4. Joint United Nations Programme on HIV/AIDS (UNAIDS). The need for routine viral load testing: Questions and answers. 2016. http://www.unaids.org/sites/default/files/media_asset//C2845_en.pdf Questions and answers. 2016

5. Haas $\mathrm{AD}$, Keiser $\mathrm{O}$, Balestre E, et al. Monitoring and switching of first-line antiretroviral therapy in adult treatment cohorts in sub-Saharan Africa: Collaborative analysis. Lancet HIV 2015;2(7):271 278

6. Stinson K, Goemaere E, Coetzee D, et al. Cohort profile: The Khayelitsha antiretroviral programme, 6. Stinson K, Goemaere E, Coetzee D, et al. Cohort profile: The Khayelitsha antiretroviral prog
Cape Town, South Africa. Int J Epidemiol 2016;46(2):e21. https://doi.org/10.1093/ije/dyw057

Cape Town, South Africa. Int J Epidemiol 2016;46(2):e21. https://doi.org/10.1093/ije/dyw057
7. Kaplan SR, Oosthuizen C, Stinson K, et al. Contemporary disengagement from antiretroviral Kaplan SR, Oosthuizen C, Stinson K, et al. Contemporary disengagement from antiretroviral
therapy in Khayelitsha, South Africa: A cohort study. PLOS Med 2017;14(11):e1002407. https://doi, therapy in Khayelitsha, South Africa: A cohort study. PLOS Med 2017;14(11):e1002407. https://doi. org/10.1371/journal.pmed.1002407

8. Drew RS, Rice B, Rüütel K, et al. HIV continuum of care in Europe and Central Asia. HIV Med 2017;18(7):490-499. https://doi.org/10.1111/hiv.12480

9. Provincial Government of the Western Cape - Department of Health. The Western Cape consolidated guidelines for HIV treatment: Prevention of mother-to-child transmission of HIV (PMTCT), children, adolescents and adults. 2016 (amended). https://www.westerncape.gov.za/sites/www.westerncape.gov. za/files/the_western_cape_consolidated_guidelines_for_hiv_treatment_29032016.pdf (accessed 21 January 2019).

10. Heekes A, Tiffin N, Dane P, et al. Self-enrolment antenatal health promotion data as an adjunct to maternal clinical information systems in the Western Cape Province of South Africa. BMJ Glob Health 2018;3(Suppl 2):e000565. https://doi.org/10.1136/bmjgh-2017-000565

11. Clouse K, Vermund SH, Maskew M, et al. Mobility and clinic switching among postpartum women considered lost to HIV Care in South Africa. J Acquir Immune Defic Syndr 2017;74(4):383-389. https://doi.org/10.1097/qai.0000000000001284

12. Phillips T, McNairy ML, Zerbe A, Myer L, Abrams EJ. Implementation and operational research: Postpartum transfer of care among HIV-infected women initiating antiretroviral therapy during pregnancy. J Acquir Immune Defic Syndr 2015;70(3):e102-e109. https://doi.org/10.1097/qai.0000000000000771

Accepted 13 August 2018. 